\title{
Design considerations for quasi-phase-matching in doubly resonant Lithium Niobate hexagonal micro-resonators
}

\author{
Tleyane J. Sono ${ }^{1,2}$, Christos Riziotis ${ }^{* 3}$, Sakellaris Mailis ${ }^{1}$, Robert W. Eason ${ }^{1}$
}

(1) University of Southampton, Optoelectronics Research Centre, Highfield, Southampton SO17 1BJ, United Kingdom

(2) Council for Scientific and Industrial Research (CSIR), Defence Peace Safety and Security, Brummeria, Pretoria 0001, South Africa

(3) National Hellenic Research Foundation, Theoretical and Physical Chemistry Institute, Photonics for Nano-applications Laboratory, Athens 11635, Greece

*Corresponding author: Christos Riziotis (Riziotis@eie.gr)

\begin{abstract}
Fabrication capabilities of high optical quality hexagonal superstructures by chemical etching of inverted ferroelectric domains in Lithium Niobate platform suggests a route for efficient implementation of compact hexagonal microcavities. Such nonlinear optical hexagonal microresonators are proposed as a platform for second harmonic generation (SHG) by the combined mechanisms of total internal reflection (TIR) and quasi-phase-matching (QPM). The proposed scheme for SHG via TIR-QPM in a hexagonal microcavity can improve the efficiency and also the compactness of SHG devices compared to traditional linear-type based devices. A simple theoretical model based on six-bounce trajectory and phase matching conditions was capable for obtaining the optimal cavity size. Furthermore numerical simulation results based on finite difference time domain (FDTD) Beam Propagation Method (BPM) analysis confirmed the solutions obtained by demonstrating resonant operation of the microcavity for the second harmonic wave produced by TIR-QPM. Design aspects, optimization issues and characteristics of the proposed nonlinear device are presented.
\end{abstract}

Keywords: Lithium Niobate, nonlinear, total-internal-reflection, quasi-phase-matching, microresonator, microcavity, second harmonic generation, simulation, optical circuits

\section{Introduction}

Current technology trends are moving photonic devices towards smaller dimensions and higher integration density, while integrated optical cavities form a characteristic technological area with increased research and technological interest. Those cavities are aimed at trapping light, ideally without any loss, until they are triggered to release the stored light out of the cavity. Practically, eliminating losses within a cavity is very hard and a more efficient optical cavity is one that can store a large fraction of the light compared to that lost during the cavity lifetime. A figure of merit, used to describe the energy stored in the cavity, relative to the energy lost by the cavity per round trip, is the quality factor $(Q)$ and therefore effective optical cavities possess high $Q$ characteristics. In optical applications, such as high speed communications systems, the integration of different optical components with strict size and packaging requirements is crucial for the development of high 
performance and practical devices. This imposes a size and compactness requirement on any optical components, such as optical cavities, as parts of high density optical integrated circuits. Thus, a high $Q$ is not enough unless the corresponding cavity is small enough to be integrated with other microoptical components and form part of a practical optical circuit. Such optical microcavities of micrometers dimensions, $[1,2]$ have attracted increased research attention as they could play a key role in the enhancement of integrated optical circuits' functionality. They are normally placed near to at least one coupling waveguide device which then allows in-coupling and out-coupling of light [3]. Microcavities have been used for various applications such as micro lasers $[4,5]$, optical filters $[6,7]$ and non-linear devices [8,9], amongst others. Light confinement and resonance within the microcavity is achieved via total internal reflection of the light beam between the guiding and the surrounding medium, and mode wavefront matching per round trip. With mature waveguide fabrication technologies available, microcavities with high modal confinements and low modal volume have started to emerge and several geometries of the cavities including microspheres, polygonal cavities [10 -13], and photonic crystal resonators have been investigated so far for a range of applications. Further to microcavities, current methods have been demonstrated for the efficient fabrication of waveguide-based devices and integrated optical circuits even in nonlinear optical platforms such as Lithium Niobate $[14,15]$ allowing thus the future integration of different optical components in single optical chips [16] for certain demanding applications.

Here, we focus on optical microcavities for nonlinear applications such as nonlinear frequency generation $[17,18]$ and more specifically on SHG devices $[19,20]$, that have been lately implemented by different fabrication architectures. Our approach here is targeted to properly utilize the signal stored at resonance within the cavity in order to enhance the efficiency of the nonlinear optical process. Nonlinear processes such as SHG have a quadratic dependence on the intensity of the fundamental input wave (FW) $\left(I_{\omega}\right)$, the total length of interaction during propagation $(l)$ and the nonlinear coefficient $\chi^{(2)}\left(\right.$ i.e. $\left.I_{2 \omega} \propto\left(I_{\omega} \chi^{(2)}\right)^{2} \operatorname{sinc}^{2}(0.5 \Delta k l)\right)$. Therefore, within a medium with high $\chi^{(2)}$, such as $\mathrm{LiNbO}_{3}[21]$ as an example, increasing $I_{\omega}$ and/or $l$ can lead to higher $I_{2 \omega}$ when phase matching is achieved (i.e $\Delta k=0$ ).

For a second order nonlinear optical process, the conversion efficiency is proportional to the square of the propagation length within the optical device. Obviously, higher efficiency for this kind of process in traditional linear devices requires longer lengths that impose a limitation for integration with any other micro-optical systems. However, high $Q$ within a microcavity implies an effective longer integration-length within a small volume. Hence multiple round trips within the microcavity can compensate longer lengths in bulky devices.

The efficiency $(\eta)$ for conversion of the FW to the second harmonic wave (SH) in a dispersive medium where $\mathrm{n}_{2 \omega} \neq \mathrm{n}_{\omega}$ is limited by the inherent phase mismatch, $k_{2 \omega} \neq 2 k_{\omega}$, between the FW and SH. Perfect phase matching (PPM), temperature tuning and quasi phase matching (QPM) techniques have been used successfully to resolve this phase matching problem [22, 23]. Another technique used to achieve the phase matching between the two waves is total internal reflection-quasi phase matching process (TIR-QPM) $[24,25,26]$, which utilizes the Fresnel phase shift between the FW and the generated SH upon total internal reflection to balance the phase shift due to dispersion $[22,27,28$, 29]. TIR-QPM has an inherent advantage as it does not require an inverted domain structure along the propagation length, as is required for QPM, and can be used throughout the transparent window 
of the material unlike the case of PPM. TIR-QPM can also be used in microcavities, where light is resonantly guided via TIR. When the light is resonantly guided, TIR-QPM can be as efficient as the perfect phase matching method.

Here we investigate the conditions for QPM for SHG, in a hexagonal microcavity made from single crystal lithium niobate [30]. The TIR-QPM process allows QPM to occur along the propagation length by balancing the dispersion phase shift with the relative Fresnel phase shift between the FW and SH induced when the two signals undergo TIR at the interface [28]. In the conventional $1^{\text {st }}$ order QPM process, the dispersion phase shift is cancelled after every coherence length $l_{c}=\pi / \Delta \mathrm{k}=$ $\lambda /\left[4\left(n_{2 \omega}-2 n_{\omega}\right)\right]$, for example, by reversing the sign of the nonlinear coefficient [22]. The hexagonal geometry was chosen due to the fact that hexagonal optical cavities of superior optical quality can be fabricated by differential etching of inverted ferroelectric domains in lithium niobate [31], as shown by figure 1 , and this platform is actually the technological drive behind the work presented here.

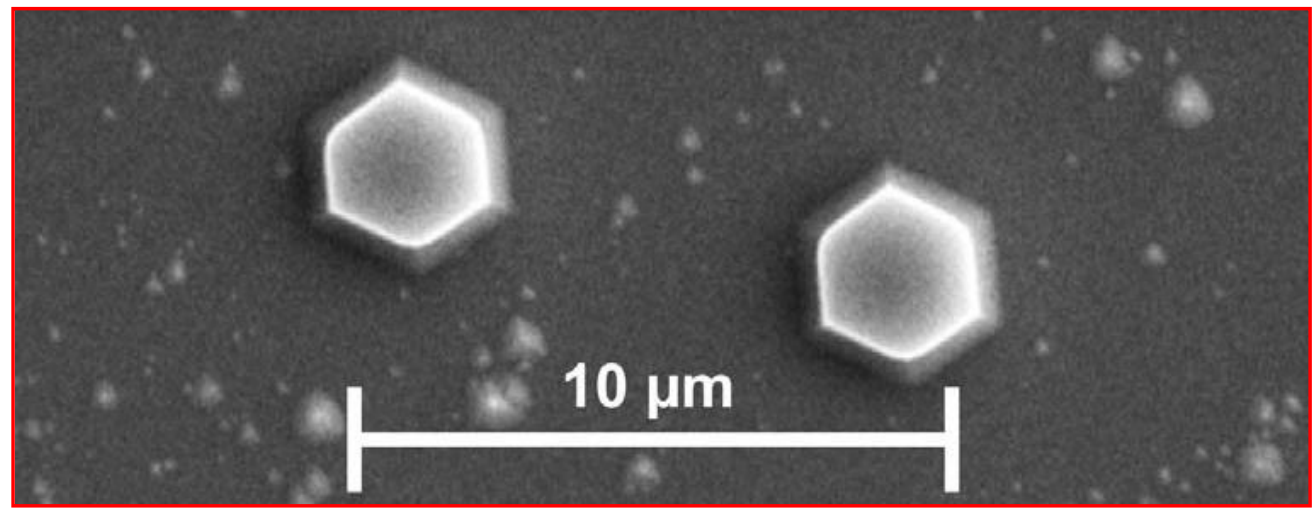

Figure 1: Hexagonal superstructure achieved by etching poled $z$-cut, $\mathrm{LiNbO}_{3}$. The $-\mathrm{z}$ face etches away while the $+z$ face remains unetched when the crystal is immersed in an $\mathrm{HF}: \mathrm{HNO}_{3}$ acid mixture

In section 2 of this paper, a theoretical analysis of TIR-QPM will be presented and compared with the perfect-phase matching PPM mechanism and with conventional QPM utilizing periodic domain inversion. Section 3 presents the model for TIR-QPM in a hexagonal cavity followed by the analytical design of a suitable hexagonal microcavity. Section 4 presents FDTD simulations for the determination of the ideal hexagonal microcavity and the estimation of SHG efficiency via the TIRQPM mechanism.

\section{Theory: Operational principle of TIR-QPM mechanism}

The intensity of the generated $\mathrm{SH}\left(\mathrm{I}_{2 \omega}\right)$, assuming no depletion of the $\mathrm{FW}$, can be written as [28]:

$$
I_{2 \omega}=\frac{8 \pi^{2} d_{e f f}^{2}}{n_{\omega}^{2} n_{2 \omega}^{2} \lambda_{\omega}^{2} \varepsilon_{0} c} I_{\omega}^{2} l_{1}^{2}\left[\frac{\sin \left(\Delta k l_{1} / 2\right)}{\Delta k l_{1} / 2}\right]^{2}
$$

where $I_{\omega}$ is the intensity of the $\mathrm{FW}, \lambda_{\omega}$ is the wavelength of the $\mathrm{FW}$ and $l_{1}$ is the interaction length between the FW and the generated SH and $d_{\text {eff }}$ is an effective nonlinear coefficient. When $l_{1}$ is of the order or longer than the coherence length $l_{c}$, the amplitude of the $\mathrm{SH}$, is modulated between zero 
and its maximum value, because of the difference in phase velocity between the SH and the FW, at every even and odd multiple of $l_{c}$ respectively, for the Non Phase Matched (NPM) case as shown in figure 2. In birefringent materials, this phase mismatch is addressed by launching a polarized FW beam(s), with an incident angle equalling the phase matching angle $\left(\theta_{m}\right)$ at which, $n_{2 \omega}\left(\theta_{m}\right)=n_{\omega}\left(\theta_{m}\right)$ leading to $\Delta \mathrm{k}=0$ and Perfect Phase Matching (PPM) [33].

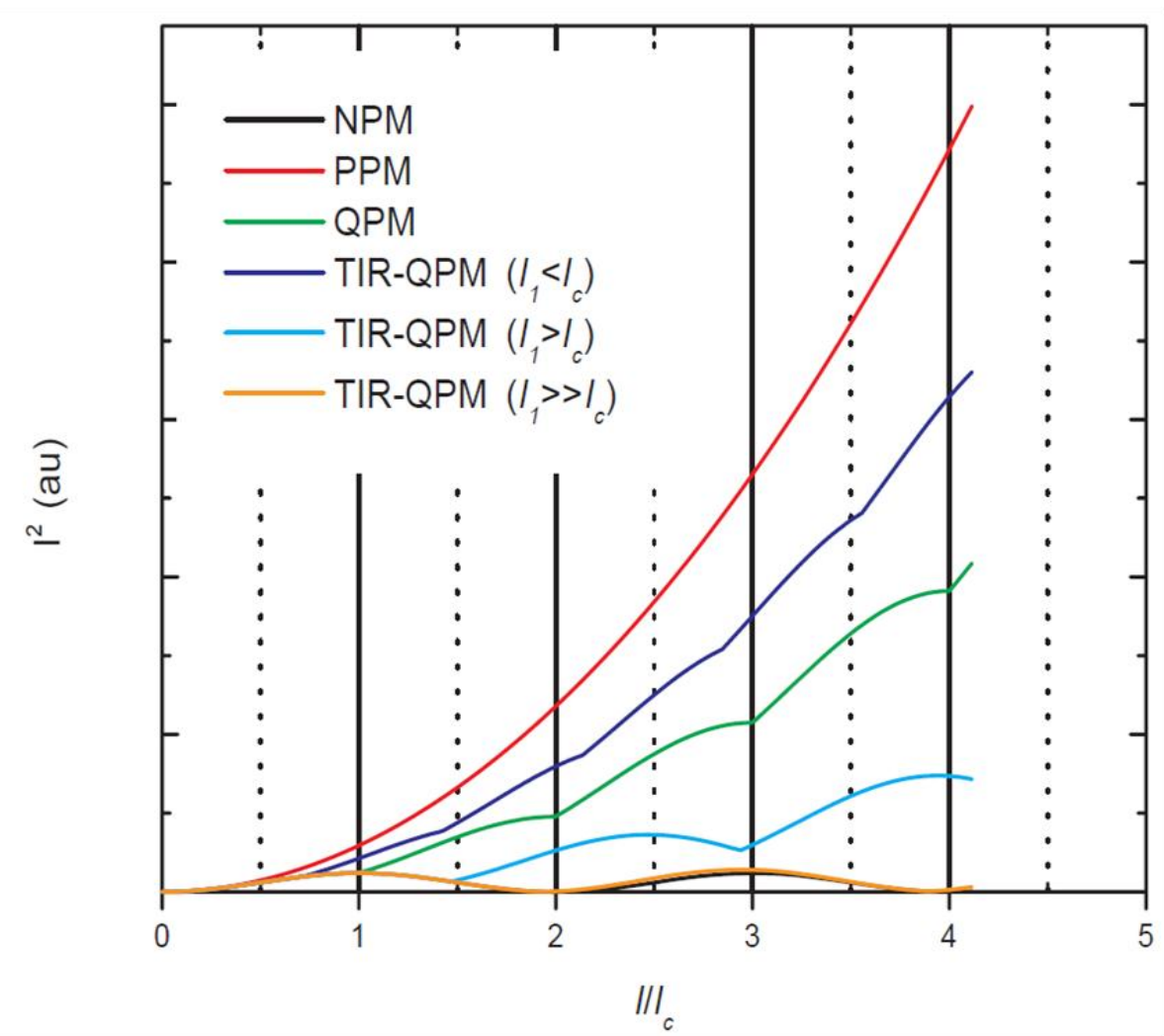

Figure 2: Growth of the SH along the propagation length within a nonlinear crystal. PPM: perfect phase matching in a single domain crystal. $I_{2 \omega}$ in this figure is normalized to that of PPM. QPM: first-order QPM in a periodically poled crystal and NPM: nonphase-matched interaction. TIR-QPM: total internal reflection-QPM. For TIR-QPM, the overall phase matching occurs not necessarily at $l_{c}$ but at a length $l_{1}$ where the Fresnel phase shift balances the dispersion phase shift. The NPM curve is not shown clearly as it largely overlap with TIR-QPM $\left(l_{1}>>l_{c}\right)$ curve.

However, the PPM method suffers from the transverse walk-off of the SH wave vector away from that of the input polarized FW if the phase matching angle is not normal to the optical axis, thus degrading the conversion efficiency. QPM is a technique commonly used to enhance the efficiency of nonlinear interactions, such as SHG, in cases where PPM cannot be applied [23]. One example of QPM is by periodic poling in $\mathrm{LiNbO}_{3}$ or KTP where the sign of the relevant nonlinear second-order coefficient $\mathrm{d}_{\mathrm{ij}}$ is inverted after each length $l_{\mathrm{c}}$ (for the case of first order QPM as shown in figure 2) to compensate for the phase shift between the FW and the SH [23]. The inversion of $\mathrm{d}_{\mathrm{ij}}$ at every $l_{\mathrm{c}}$ prevents the down conversion of SH into FW at every odd number of $l_{\mathrm{c}}$ and enables the growth of $\mathrm{SH}$. However the QPM technique has several advantages over the PPM technique as in QPM, the whole window of optical transparency and all corresponding nonlinear coefficients of a material can be used for effective nonlinear interaction provided that the desired QPM period $(\Lambda)$ can be achieved. Also, QPM allows the use of the largest nonlinear coefficient which for $\mathrm{LiNbO}_{3}$ is $\mathrm{d}_{33}$, as an example, since the input incident angle of the $\mathrm{FW}$ is not limited to $\theta_{\mathrm{m}}$. 
However, in the QPM process, $\Lambda \propto \lambda$ where $\Lambda$ is the period of the inverted domain pattern, which implies the requirement of short periods for low values of wavelength which are not always implementable via conventional methods. Another way to achieve QPM is by utilizing the relative Fresnel phase shift $\left(\Delta \Phi_{\mathrm{F}}\right)$ given in equation (2) between the $\mathrm{FW}$ and the induced $\mathrm{SH}$, that occurs upon TIR of the two waves at a cavity interface after propagating for a length $l_{1}$, to compensate for the dispersion phase mismatch $\left(\Delta \mathrm{k} \cdot l_{1}\right)[22,28]$. In this method, referred to as "TIR-QPM", both the FW and the induced SH are made to be reflected via TIR on a surface for the case of propagation in a parallel sided plate. This way FW and SH experience a relative phase difference that can cancel that due to dispersion. The relative Fresnel phase shift is given as:

$$
\Delta \Phi_{F}=\Phi_{\gamma}(2 \omega)-2 \Phi_{\gamma}(\omega)
$$

where $\Phi_{\gamma}(2 \omega)$ and $\Phi_{\gamma}(\omega)$ are the Fresnel phase shifts of the SH and FW, respectively, which occurs after each TIR. Parameter $\gamma$ is either $p$ or $s$ depending on the polarization of both FW and SH. The effective nonlinear coefficient $\left(\mathrm{d}_{\text {eff }}\right)$ can also change sign following reflection depending on the input/output polarizations of both FW and SH relative to the crystal symmetry. The possible change of sign of $d_{\text {eff }}$ results in an extra phase shift $(\xi \pi)$, with $\xi$ being either 1 or 0 , respectively. For values of $\theta$ above the critical angle $\theta_{c}, \Delta \Phi_{\mathrm{F}}$ can take any value from 0 and $2 \pi$, as $\theta$ increases towards $90^{\circ}$. Thus, the combination of $\Delta \Phi_{\mathrm{F}}$ and $\xi \pi$ can, in general, compensate for any dispersion phase shifts and allows for a more flexible choice of the propagation length, between adjacent reflections, as compared to the periodic poling case where the $2 l_{c}$ period constraint is strict. The global phase shift $\phi$, between the FW and SH during TIR, is therefore the combination of all these phase shifts and is given in the following equation [28].

$$
\phi=\Delta k l_{1}+\Delta \Phi_{F}+\xi \pi=2 \pi m, \quad m=0,1,2, \ldots
$$

For a given $\omega, \theta$ and set input/output polarization state of the waves, the condition in equation (3) is only possible at certain values of $l_{1}$. For a given reflectivity coefficient $r$ for each total internal reflection bounce, the intensity of the generated $\mathrm{SH}\left(I_{2 \omega}\right)$, can be written as [27]:

$$
I_{2 \omega}=\frac{8 \pi^{2} d_{e f f}^{2}}{n_{\omega}^{2} n_{2 \omega}^{2} \lambda_{\omega}^{2} \varepsilon_{0} c} I_{\omega}^{2}\left(M l_{1}\right)^{2}\left[\frac{\sin \left(\Delta k l_{1} / 2\right)}{\Delta k l_{1} / 2}\right]^{2} \frac{r^{M}}{M^{2}} \frac{1-2 \sqrt{r^{M}} \cos (M \phi)+r^{M}}{1-2 \sqrt{r} \cos (\phi)+r}
$$

while for no-reflectivity-induced loss ( $r=100 \%)$ it can be expressed in the simplified form:

$$
I_{2 \omega}=\frac{8 \pi^{2} d_{e f f}^{2}}{n_{\omega}^{2} n_{2 \omega}^{2} \lambda_{\omega}^{2} \varepsilon_{0} c} I_{\omega}^{2}\left(M l_{1}\right)^{2}\left[\frac{\sin \left(\Delta k l_{1} / 2\right)}{\Delta k l_{1} / 2}\right]^{2} \times\left[\frac{\sin (M \phi / 2)}{M \sin (\phi / 2)}\right]^{2}
$$

where $I_{\omega}$ is the intensity of the $\mathrm{FW}, \lambda_{\omega}$ is the wavelength of the $\mathrm{FW}$ and $\mathrm{M}$ is the number of zig-zag paths or single bounces. In equation (5), the first trigonometric part represents a parametric conversion on each path, $l_{1}$, travelled by the mode between two reflections, while the second trigonometric part represents the interaction of all input and generated fields after each reflection. 
For the case when $l_{1}$ is exactly an odd number of $l_{\mathrm{c}}$, the combination of $\Delta \Phi_{\mathrm{F}}$ and $\xi \pi$ has to sum up to $\pi$ for QPM to occur, in which case both factors in equation (5) are maximized [28]. This case is referred as resonant TIR-QPM and is found to be practically more efficient as compared to the nonresonant case. Figure 2 compares the efficiency of the PPM, QPM and TIR-QPM methods.

The other advantage of TIR-QPM is that $l_{1}$ can take up any values but not limited to the integral multiple of $l_{c}$. For the case where $l_{c}>l_{1}$ the efficiency of the TIR-QPM is ideally comparable to that of the higher order QPM process while for $l_{c}<l_{1}$ the efficiency approaches that of PPM as shown in figure 2. For large values of $l_{1}$ relative to the $l_{c}$, the TIR-QPM efficiency is lower to that of NPM. The limitation to this ideal case is mostly due to the reflection losses that occur at each reflection. In figure 2 , three values of $l_{1}=1.72,7.35,12.71 \mu \mathrm{m}\left(l_{\mathrm{c}}=2.39 \mu \mathrm{m}\right.$ for $\left.\lambda=955 \mathrm{~nm}\right)$ were used and the reflection coefficients were set to be $100 \%$.

Intensity $I_{\omega}$ in Eq. (1) is defined at the input and it stays at that value throughout $l_{1}$, so the value $I_{2 \omega}$ increased mainly due to $l_{1}$. Within a single-resonant microcavity where FW resonates, $I_{\omega}$ increases after each round trip until the steady state condition is met. Basically, $I_{\omega}$ is amplified until steady state. Therefore, $I_{2 \omega}$, increases not only due to increment of $l_{1}$ but also due to the amplification of $I_{\omega}$. In a doubly-resonant microcavity where $\mathrm{SH}$ also resonate, $I_{2 \omega}$ is further amplified. Since the idea is to use the stored and cavity-enhanced FW (by the cavity) to increase the efficiency of the SHG, the ideal cavity of size $\alpha_{s}$ is the cavity that can simultaneously accommodate and sustain the resonance of both FW and SH (doubly resonant).

\section{TIR-QPM Model in Hexagonal Cavity}

Previous published works have been focussed on the study of waveguiding in hexagonal microcavities [34, 35, 36], where the incident angle dependence of the optical power build-up in a hexagonal cavity was investigated showing an increase of number of cavity round trips resulting in sharp resonance for a reflection angle of $60^{\circ}$. Only at this angle can the length between subsequent reflections be constant and support long lived resonances with high $Q$. This restriction is also critical for QPM operation inside the cavity, as will be demonstrated here by two-dimensional (2D) FDTD (RSOFT Simulation Software) simulation studies. In the FDTD simulation the permittivity tensor of Lithium Niobate was fully considered as it was included as a built in option in the simulation software. Input field FW is being launched within the cavity in order to maintain the $60^{\circ}$ reflection angle [34]. In our analysis we followed this launching approach instead of the side evanescent wave coupling in order to focus on the operational principle rather than on other technical issues such as the design of side coupling sections. At this reflection angle, we avoid open-loop trajectories which although they also lead to cavity resonance modes (provided they are waterfront-matched after each cavity round-trip), they however have a short cavity life-time [28, 35]. A reflection angle of $60^{\circ}$ also guarantees that the length $\left(l_{1}\right)$ between subsequent TIRs in the regular hexagonal cavity is equal, which simplifies the expression for phase compensation.

The first step of the analysis will be to determine by a simplified but effective analytical model the optimal cavity size which supports both a resonance for the FW and provides TIR-QPM for second harmonic generation. 


\subsection{Analytical Design}

First, we consider the requirement for the FW to resonate in a hexagonal microcavity. The angle $(\theta)$ of incidence at each reflective surface within this cavity is set at $60^{\circ}$ and is fixed at this value for the entire model. Our model is based on a $\mathrm{LiNbO}_{3}$ crystal as a bulk platform and, since this crystal is both uniaxial and dispersive, we have to pay attention to the polarization of the FW and use the corresponding refractive index.

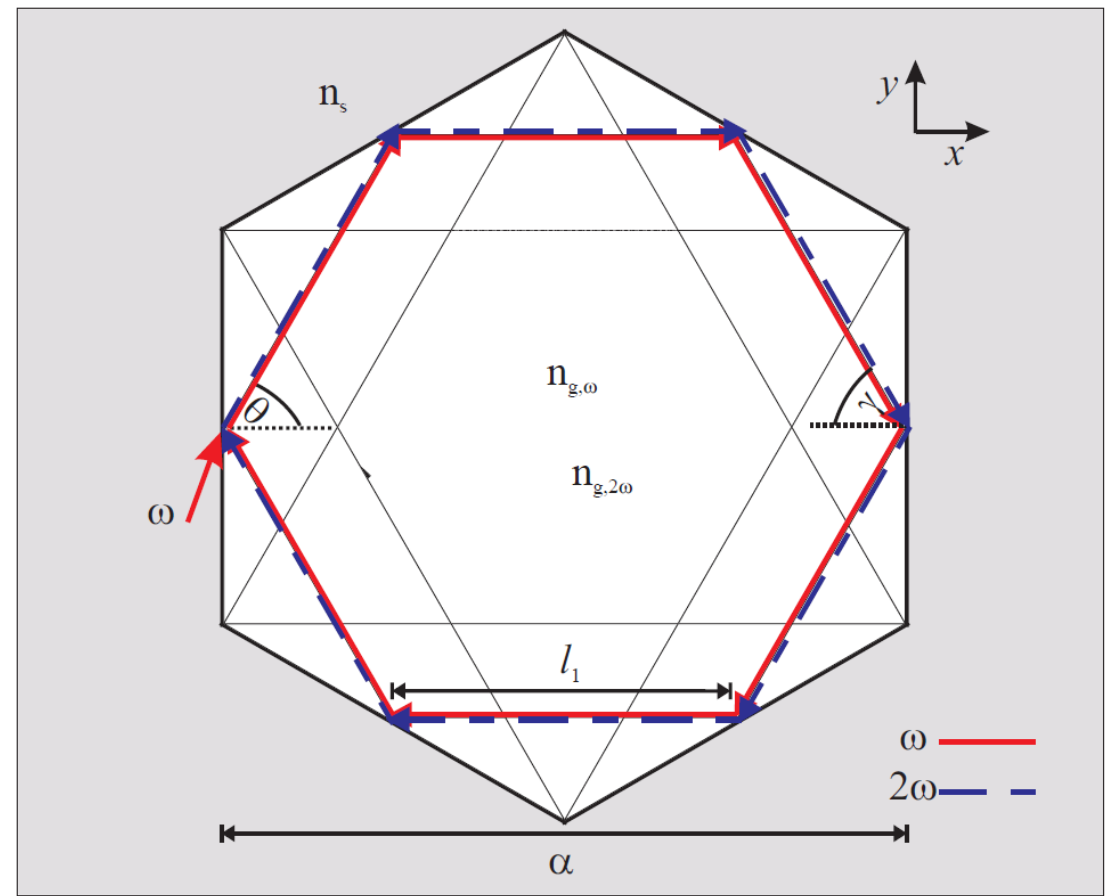

Figure 3: Hexagonal cavity showing schematic resonance propagation of both the FW and SH.

We consider here s-polarization for the FW, assuming that the c-axis of the crystal is perpendicular to the plane of the hexagonal cavity hence it experiences the extraordinary refractive index $\left(n_{\omega}\right)$. For dispersion calculation, the Sellmeier equations were used to determine the corresponding indices of refraction. For a given free space wavelength $\lambda_{0}$, resonance of a given mode, defined by $\theta$ inside the hexagonal cavity, has to satisfy the wavefront matching condition given below $[35,36]$ :

$$
k_{o} n_{g} L\left(\theta_{\omega}\right)+3 \Phi_{F}(\theta)+3 \Phi_{F}\left(120^{\circ}-\theta\right)=2 \pi m_{\omega}, \quad m_{\omega}=0,1,2,3
$$

where $k_{0}$ is the free space propagation constant and the integer $m_{\omega}$ is the azimuthal mode order, of a six-bounce wavefront-matched cavity mode travelling over a single cavity-round trip of length $L(\theta)=3 \alpha \sin \left(30^{\circ}+\theta\right)$ within a cavity with size $\alpha$.

As far as the resonance of the FW is concerned, what remains is to find the correct cavity size $\alpha_{\omega}=L\left(60^{\circ}\right) / 3$ at the given wavelength, such that the left side of equation (6) equals to an integer multiple of $2 \pi$. Since $\cos (m \pi)= \pm 1$, for integer values of $m$, the resonance condition in equation (6) for FW can be rewritten as: 


$$
f_{\omega}=\cos \left(\frac{1}{2}\left[k_{o} n_{e, \omega} L_{\alpha_{\omega}}+6 \Phi_{s, \omega}\right]\right)=\cos \left(m_{\omega} \pi\right)= \pm 1
$$

Likewise, the resonance condition for the SH can be written as:

$$
f_{2 \omega}=\cos \left(\frac{1}{2}\left[k_{o} n_{e, 2 \omega} L_{\alpha_{2 \omega}}+6 \Phi_{s, 2 \omega}\right]\right)=\cos \left(m_{2 \omega} \pi\right)= \pm 1
$$

where $\alpha_{2 \omega}$ is the resonating cavity size for SH while $L_{\alpha_{\omega}}, L_{\alpha_{2 \omega}}$ represent the round cavity length corresponding to optimum cavity sizes $\alpha_{\omega}$ and $\alpha_{2 \omega}$ by assuming $\theta=60^{\circ}$. For a doubly resonating cavity size for both the FW and the SH, equations (7-8) must be satisfied simultaneously, in which case $\alpha_{\omega}=$ $\alpha_{2 \omega}$. Also for the TIR-QPM process, the global phase shift in equation (3) can be expressed as:

$$
f_{\phi}=\cos \left(\frac{1}{2}\left[\Delta k l_{1}\left(\alpha_{\phi}\right)+\Delta \Phi_{F}+\xi \pi\right]\right)=\cos \left(m_{\phi} \pi\right)= \pm 1
$$

where $\alpha_{\phi}$ is the cavity size at which the dispersion phase shift will be balanced by the Fresnel phase shift. The ideal cavity size is $\alpha_{m}=\alpha_{\omega}=\alpha_{2 \omega}=\alpha_{\phi}$. Within the ideal cavity, all the factors in equations (79) have values of \pm 1 and so their products as described by the resulting equation (10) that combines the above three equations:

$$
f=f_{\omega}\left(\alpha_{m}\right) \times f_{2 \omega}\left(\alpha_{m}\right) \times f_{\phi}\left(\alpha_{m}\right)= \pm 1
$$

Figure 4 shows the variation of the functions $f_{\omega}, f_{2 \omega} f_{\phi}$ and $f$ in equations (7-9) for the s-polarized FW and $\mathrm{SH}$ in z-cut $\mathrm{LiNbO}_{3}$ at $\lambda=0.959 \mu \mathrm{m}$. Each of these functions has maximum values of \pm 1 , at which resonance of the corresponding wave is achieved. For example at $\alpha=2.907 \mu \mathrm{m}, f_{2 \omega}=-1$ while $f_{\omega} \approx 0.1$ which means SH will resonate in the cavity of this size while FW will not. In calculating equations (79) an s-polarization was selected so to make it possible for use of largest of the nonlinear coefficients $\left(d_{33}\right)$ of the $z-$ cut $\mathrm{LiNbO}_{3}$ to be used for generation of $\mathrm{SH}$. 


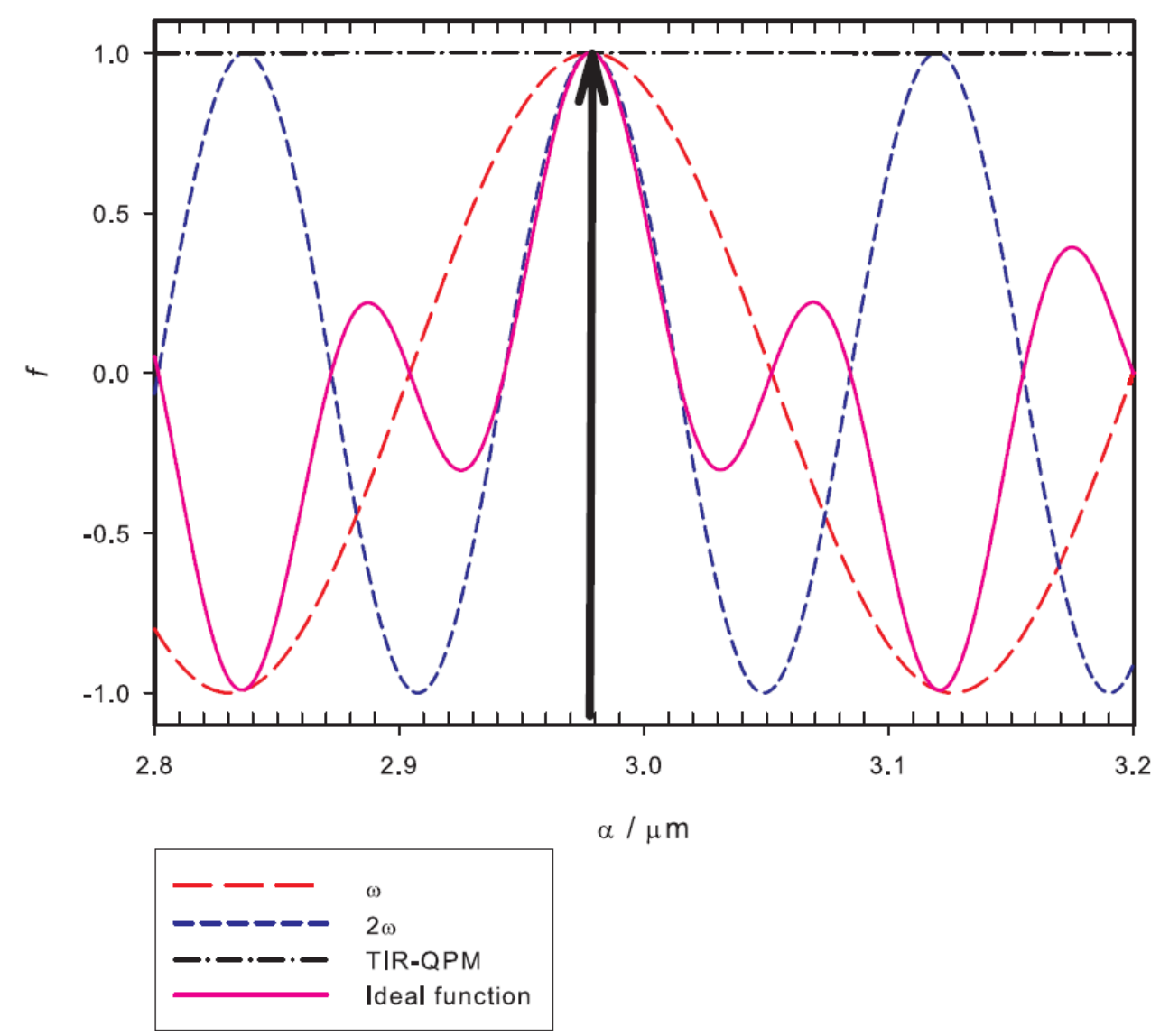

Figure 4: Plot showing the dependence of the cavity resonances of the FW, SH, TIR-QPM condition and the ideal condition on the cavity of dimension $\alpha$. The ideal cavity size is therefore $\alpha_{m}=2.98 \mu \mathrm{m}$ for $\lambda=0.959 \mu \mathrm{m}$.

Figure 4 displays six values of $\alpha_{2 \omega}$ shown by \pm 1 values of the blue dashed curve, three values of $\alpha_{\omega}$ shown by \pm 1 values of the red long-dash curve, from where it is obtained that the optimum cavity size is $\alpha_{m}=2.98 \mu \mathrm{m}$, since is satisfied $\alpha_{2 \omega}=\alpha_{\omega}=\alpha_{\phi}$. Figure 5 shows the variation of the combined function $f$ and also the function $f_{\phi}$ corresponding to global phase shift factor, against the cavity size, which shows the locations of other possible cavity dimensions $\alpha_{m}$ at $\alpha_{m 1}=2.98 \mu m, \alpha_{m 2}=12.73 \mu m$, and $\alpha_{\mathrm{m} 3}=22.35 \mu \mathrm{m}$, for $\lambda=0.959 \mu \mathrm{m}$.

The value of $\alpha_{m}$ is determined from the constraints formulated above and will be used in the next section as a guide to locate the ideal cavity size from the FDTD simulation results. Also, the cavity characteristics, i.e repetition of the solution of the FW in figure 4, will be used as a benchmark to validate the FDTD solutions. 


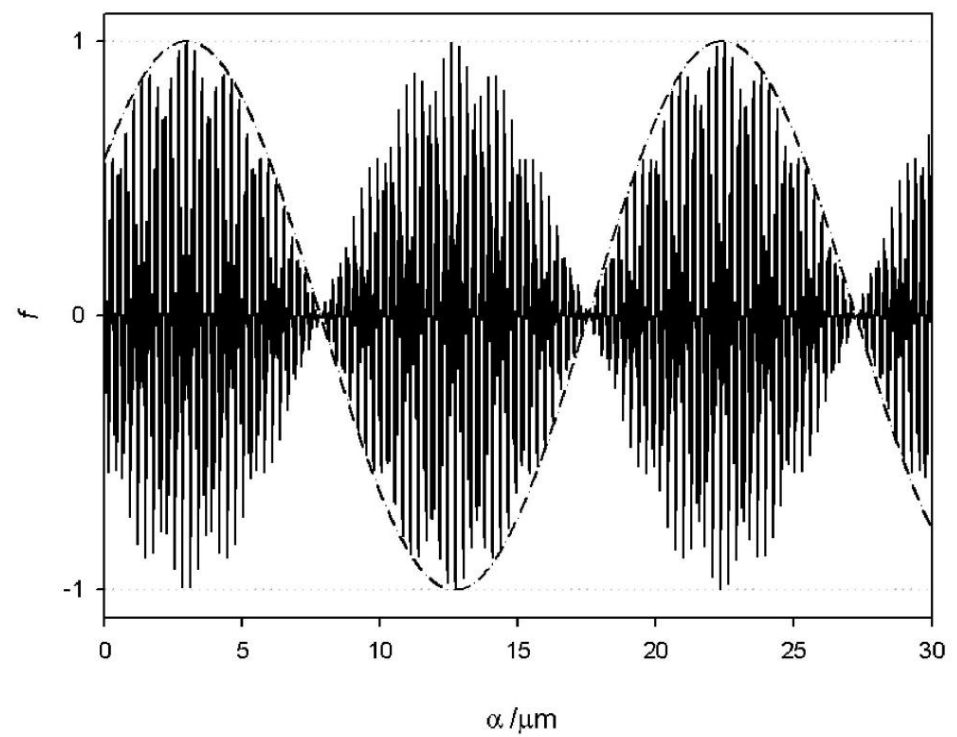

Figure 5: Plot showing the dependence of the combined function $f$ versus the cavity size. The dashed line represents the factor $f_{\phi}$ corresponding to global phase shift $\phi$.

\section{Simulation Results}

FDTD simulations were performed to investigate the response of an ideal hexagonal microcavity for both FW and SH waves. Emphasis was given to the individual propagation of the FW and SH within the microcavity. It has to be stressed that the SH here does not refer to the directly calculated second harmonic signal generated by the FW, but simply to a mode launched at half the wavelength of the FW. The evolution of this mode within the microcavity will have similar properties to the $\mathrm{SH}$ generated by FW. Thereafter we incorporate the propagation characteristics of the cavity for both FW and SH to estimate the SHG efficiency. The refractive index of the cavity at FW and SH corresponds to the extraordinary refractive index of congruent $\mathrm{LiNbO}_{3}$, as given by the Sellmeier equation for $\lambda$ and $\lambda / 2$. The polarization of the launched mode was set to TE (E-field is perpendicular to the cavity plane).

\subsection{Determination of the ideal cavity size using FDTD simulation}

Using the FDTD simulation software we will calculate a suitable resonator size (a value of $\alpha_{m}$ ) for simultaneous resonance of $\mathrm{FW}$ and $\mathrm{SH}$. This more rigorous numerical approach will be also used to validate the optimum resonator size that was calculated earlier using the simplified analytical model.

Continuous simulation runs of the cavity for a range of $\alpha_{m}$ and for the two different resonant modes FW and SH were performed. From figure 6 we identify the optimum parameter $\alpha$ at the value 2.98 $\mu \mathrm{m}$ where both $\mathrm{FW}$ and $\mathrm{SH}$ exhibit their maxima. Within the convergence range for both $\mathrm{FW}$ and $\mathrm{SH}$ simulation, the values of $\alpha_{m}$ approach their calculated value. This procedure was used to determine the value of $\alpha_{\mathrm{m}} \approx 2.98 \mu \mathrm{m}$ in figure 4 from the TIR-QPM model. For a given wavelength $\lambda$, sustained cavity modes have similar properties. Hence, a full study of the cavity at the FW and SH can be achieved by sustained cavity modes of the individual resonances. The $\mathrm{SH}$ is estimated based on the FW power amplification and on the Q-factors of both the FW and SH launched individually in the cavity. 


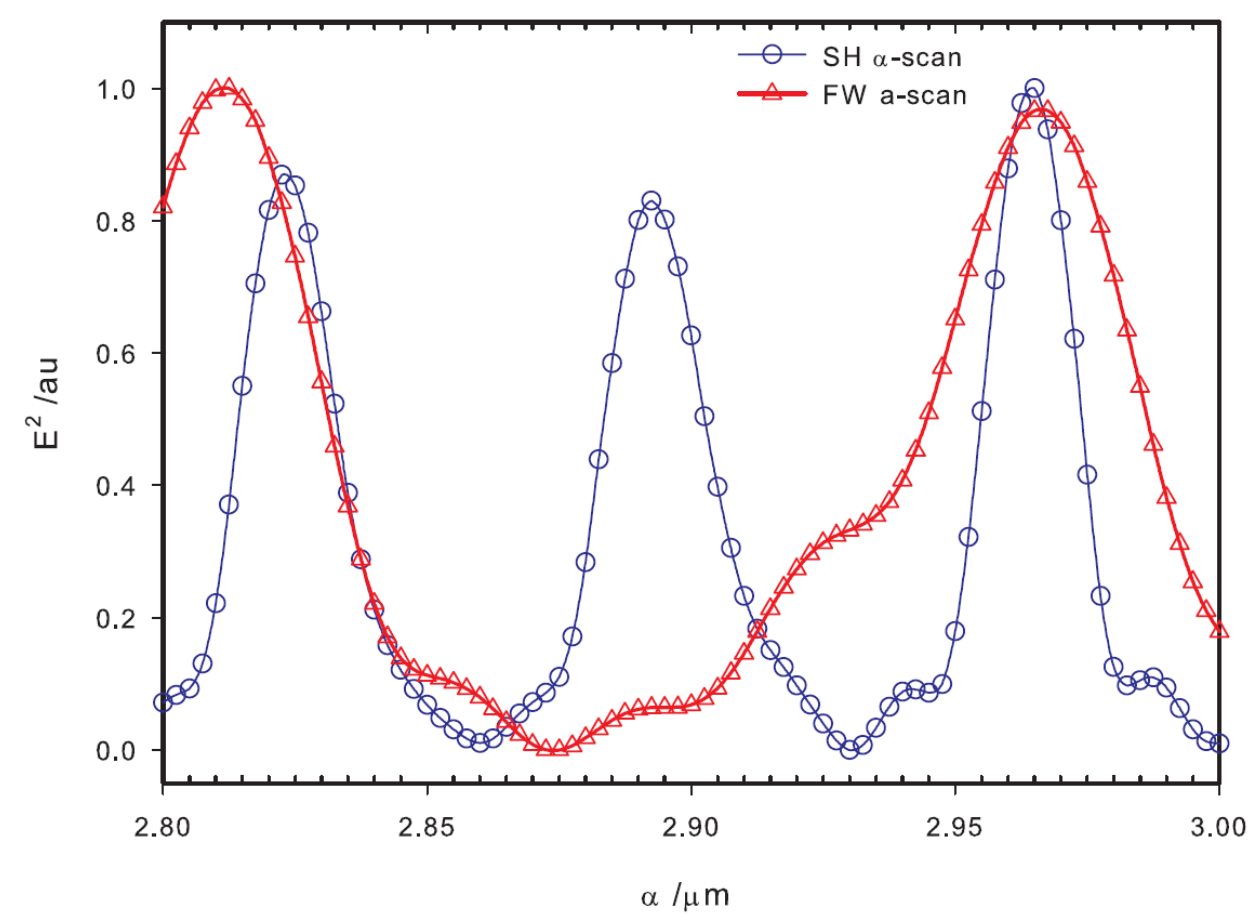

Figure 6: FDTD simulation showing the optimum cavity parameter $\alpha$ for simultaneous FW and SH resonance. The continuous scanning of the cavity gives the value $\alpha_{m 1}=2.98 \mu \mathrm{m}$ where $\alpha_{2 \omega}=\alpha_{\omega}$.

Figure 6 shows the scan of the FW (SH) for $\lambda_{\omega}=959 \mathrm{~nm}\left(\lambda_{2 \omega}=\lambda_{\omega} / 2\right)$. The refractive indices of the FW and the SH were set to $n_{e, \omega}=2.1627$ and $n_{e, 2 \omega}=2.2632$, respectively. From the above defined TIR-QPM model it was shown that an ideal cavity size can be located for $f\left(\alpha_{m}\right)= \pm 1$, at a given wavelength. In figure 6 , the ideal cavity size corresponds to the value $\alpha_{\mathrm{m}} \approx 2.98 \mu \mathrm{m}$ where $f_{\omega} \times f_{2 \omega} \approx 1$.

The determination of the ideal cavity size based on the analytical simplified TIR-QPM model shown in figure 4 is justified by comparing this figure with figure 6 obtained from the FDTD simulation. From figure 4 , the period of the FW resonating cavity size, denoted by the separation between the adjacent absolute maxima $f_{\omega}= \pm 1$ is $140 \mathrm{~nm}$, which is similar to the separation of the FW modes in figure 6 . The same applies for the $\mathrm{SH}$, where the period is about half that of the FW. This direct comparison between the FDTD simulation of the scan results and the TIR-QPM model results validates our theoretical approach in terms of the six-bounce propagation trajectory in a hexagonal microcavity.

\subsection{Steady-state Cavity Response}

In order to obtain the cavity response on resonance, the steady-state simulations were performed at the resonance wavelength. This was achieved by launching the fundamental mode and measuring the cavity response as a function of time until the cavity response reached the steady state, at which point the cavity loss per round trip and the cavity amplification per round trip are equal. A moderate cavity was used to minimize the cavity losses due to the sharp corners and also to minimize the large computational time required by a large cavity size. For $\lambda=955 \mathrm{~nm}$, the corresponding suitable ideal cavity size is found to be $\alpha_{\mathrm{m} 2}=13.95 \mu \mathrm{m}$ which is equivalent to a solution for $\lambda=959 \mathrm{~nm}$ in figure 5 . Here, the value of $\alpha_{\mathrm{m} 2}$ is for $\lambda=955 \mathrm{~nm}$ instead of $\lambda=959 \mathrm{~nm}$ due to the fact that $f_{\mathrm{m}}(955)$ was closer to a unity value than $f_{\mathrm{m}}$ (959). 
Figure 7 shows power build-up for FW and SH corresponding to $\lambda$ and $\lambda / 2$ wavelength assuming a perfect hexagonal cavity. For this ideal cavity size, the cavity has a higher $Q$ for $\lambda / 2$ than for $\lambda$, as shown by maximum number of round trips $\left(\mathrm{N}_{\max }\right)$ and the corresponding time before steady-state. For FW, the amplification of the power due to cavity resonance and power build-up stops at about 5 ps which equate to about $\mathrm{N}_{\text {max,FW }}=13$ round trips. At steady state, it is understood that the cavity gain per round trip is equal to the cavity loss per round trip and therefore there is no more amplification of the beam. The FW reaches steady state in about 5 ps while the SH beam would take about 12 ps which corresponds to $\mathrm{N}_{\mathrm{max}, \mathrm{SH}}=33$ roundtrips.

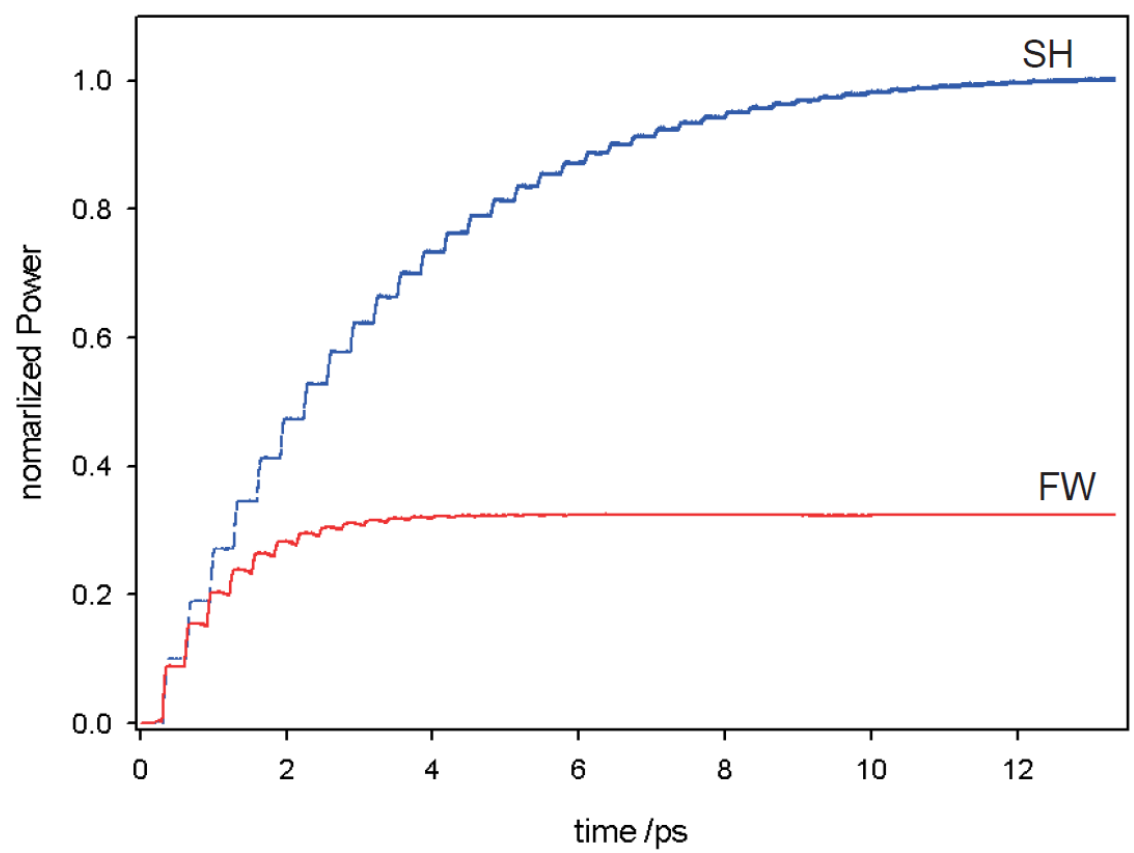

Figure 7: The cavity response for both $\mathrm{FW}$ and $\mathrm{SH}$ at a moderate ideal cavity size of $\alpha_{\mathrm{m} 2}=13.95 \mu \mathrm{m}$. The power output is normalized to that of $\mathrm{SH}$ at steady state.

For the FDTD simulation a monitor functionality, common in such simulations, was employed and the monitor's surface was virtually located inside the cavity bisecting one of the sharp corners of the hexagon. The monitor allows for the field components, power or energy density to be detected and monitored versus elapsed time at that position in the cavity. The input beam is launched away from the monitor in a way to be reflected at the wall of the hexagon at the desired angle and to be detected at the monitor only after the completion of six bounces or a round trip. The step like behavior therefore, shows the build up at the monitor per total round trips or the corresponding elapsed time.

Figure 8 shows an exponential growth curve fitted to the cavity power profile of FW in figure 7 and the following power profile was determined versus the count of round trips $\mathrm{N}$ :

$$
P_{\omega}(N)=P_{\omega, \max }(1-\exp (-b N))
$$

where $\mathrm{P}_{\omega, \max }$ is the maximum amplified power of the $\mathrm{FW}$ while $\mathrm{b}=0.330$ and represents the cavity loss per round trip. The FW amplification for the hexagonal cavity modelled in figure 7 follows this 
equation (11). By using the values in figure 7 for $\mathrm{N}_{\max }$ and amplification values for FW given by equation (11) the SHG output from the cavity is estimated in the next section.

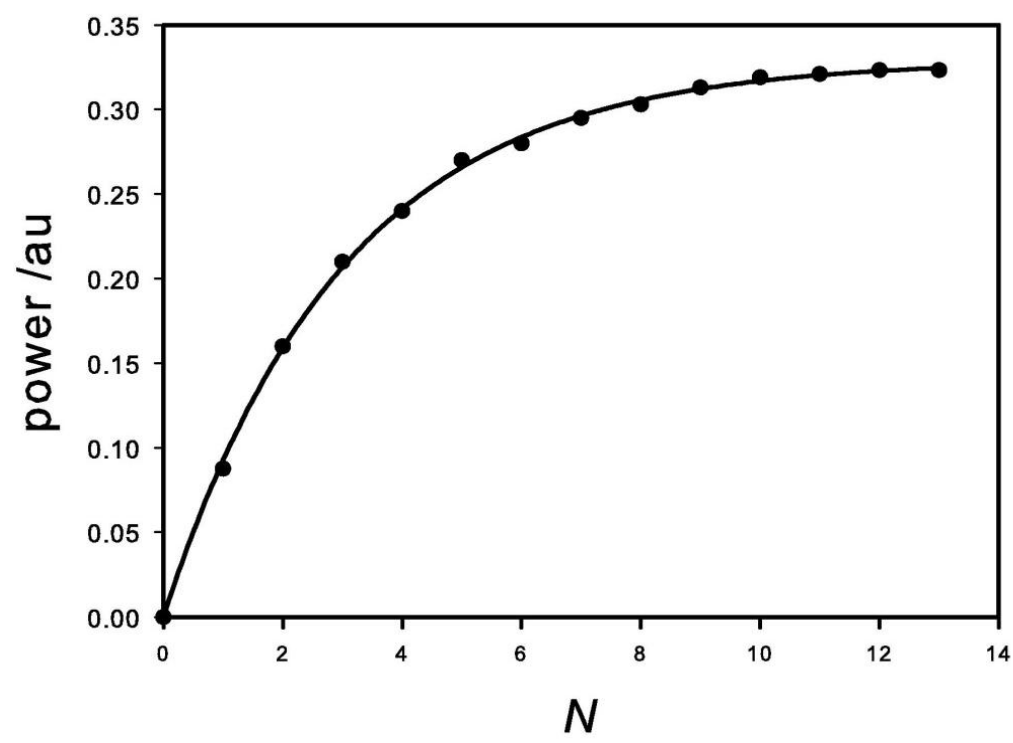

Figure 8: A curve fitting to the power evolution for the FW versus the $\mathrm{N}$ number of round trips, within a hexagonal microcavity, to determine the cavity loss per round trip $b$ for an ideal cavity size of $\alpha_{\mathrm{m} 2}=13.95 \mu \mathrm{m}$, and $b=0.33$.

\subsection{SHG efficiency via TIR-QPM in a hexagonal cavity}

Due to limitations in the capabilities of the FDTD simulation software it was not possible the direct calculation of SHG conversion yield within the cavity. Therefore the SHG conversion yield was estimated only indirectly by using the TIR-QPM model, together with values obtained from the simulation to estimate the SHG efficiency. We reasonably assume that the ideal cavity size obtained from the FDTD simulation has equivalent properties to those obtained from the model above. Our assumption has been justified above by comparing the cavity size, as this was obtained by the FDTD simulation, and the one obtained by using the model of six-reflection wavefront matched trajectories. It was found that the separation between the -1 and +1 solution in the TIR model is similar to the peaks' separation obtained in Figure 6. Also as described in the previous section, the obtained fitting curve allows the determination of the cavity loss per round trip. Based on the above assumptions we can then use the cavity $Q$ factor and the number of round trips for both FW and SH together with the value of the power for each of the FW round trips up until the steady state obtained from the FDTD simulation to estimate the SH growth in the cavity.

For the SHG efficiency estimation, we would need to know the cavity $Q$ factor for both the FW and $\mathrm{SH}$ and also the cavity amplification for FW. The cavity $\mathrm{Q}$ factor for $\mathrm{SH}$, which is different from the $\mathrm{FW}$, defines the maximum number of the $\mathrm{SH}$ round trips $\left(\mathrm{N}_{\mathrm{max}, \mathrm{SH}}\right)$. We will assume that, when the $\mathrm{FW}$ has reached the steady state $\left(\mathrm{N}_{\text {max,FW }}\right)$, there will be no more $\mathrm{SH}$ build-up within the cavity once the $\mathrm{N}_{\text {max }, \mathrm{SH}}$ is reached. The SH will grow in the cavity until its growth is balanced by its loss, at which point the $\mathrm{SH}$ output will be constant for the case where $\mathrm{Q}_{\mathrm{FW}}<\mathrm{Q}_{\mathrm{SH}}$.

SH growth is attributed to the combination of two mechanisms: firstly, the conversion growth due to the presence of the FW over the entire length of the FW propagation with the cavity and secondly, 
the gain growth of the $\mathrm{SH}$ as it builds up within the cavity. The latter growth contribution is due to the fact that the cavity resonate both the FW and SH. If, for example, the cavity did not resonate the $\mathrm{SH}$, the only SH growth mechanism would be that due to the presence of the FW similar to the normal QPM. This individual conversion growth mechanism, must lead to high SHG efficiency per volume of the hexagon via the quadratic dependence of the $\mathrm{SH}$ on the resonated $\mathrm{FW}$. In other words, this conversion mechanism will be the dominant one, however it will not be effective on its own as most of the SH signal will be radiated out if the latter signal itself is not resonated.

Equation (5) was then used to estimate the SH build-up within the cavity until it reaches the steady state, determined by $\mathrm{N}_{\max , \mathrm{SH}}$. The angle of incidence was fixed at $60^{\circ}$ and only the six-bounce trajectory per round trip, with equal propagation length between adjacent reflections was considered. This is because, though these modes are wavefront matched, the SHG will not be the same when the lengths per bounce $\left(l_{1}\right)$ are not the same. The longer path length between sequential reflections will undergo the TIR-QPM later than the shorter path ones, leading to different SHG efficiency per length.

Figure 9 shows the estimation of the growth of the SH in a regular hexagonal cavity (with no corners rounding) via the use of equation (4) for different values of the reflection coefficient ( $r$ ).

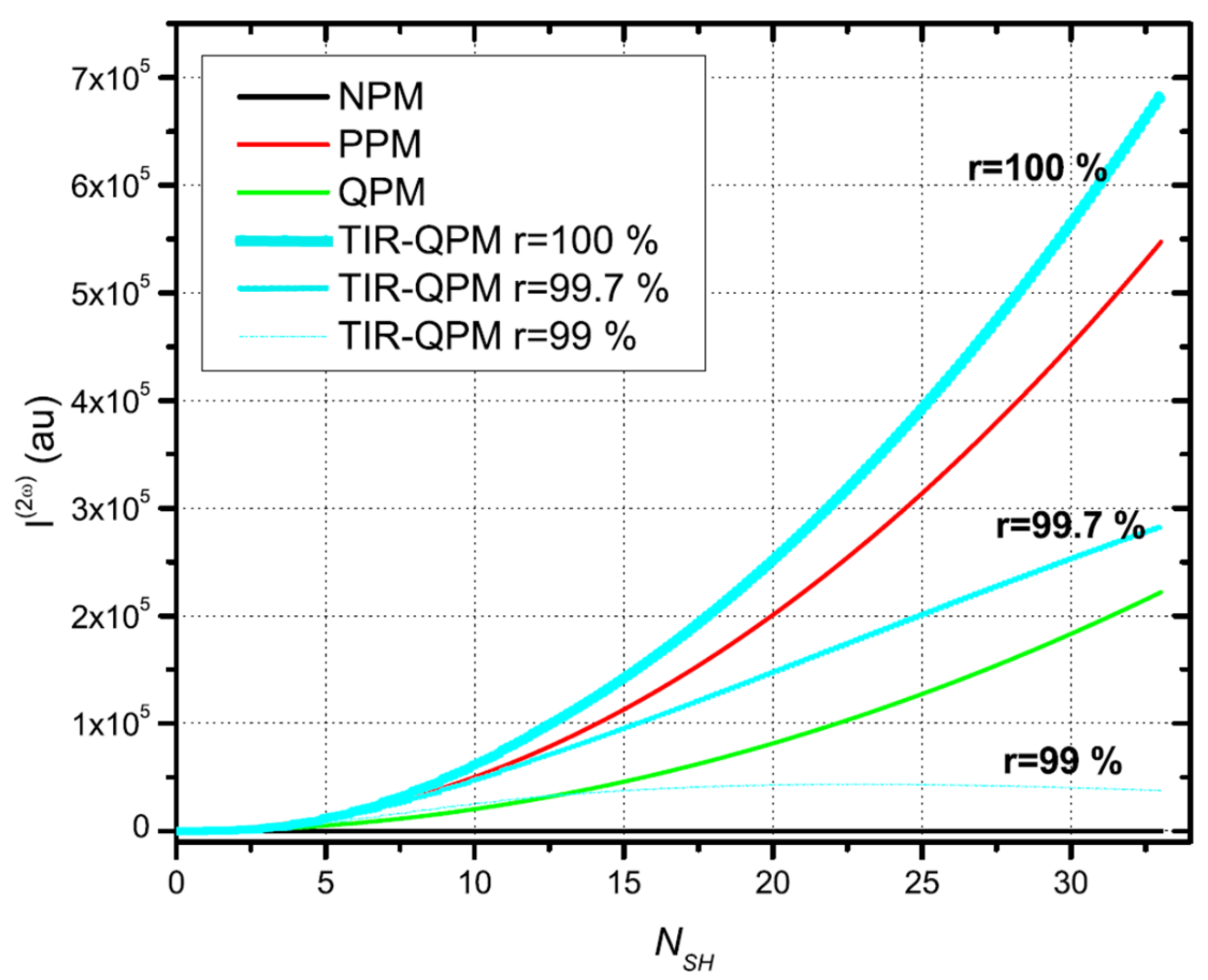

Figure 9: Estimated power of SH growth (versus the number of round trips) via TIR-QPM for a regular hexagon with no rounding, for $\lambda=0.955 \mu \mathrm{m}$ with $\alpha_{\mathrm{m} 2}=13.95 \mu \mathrm{m}$ and for different reflectivities $r$. The total length of a linear devices used to estimate the SH growth for PPM and QPM is $3 \mathrm{~N} \alpha=1.25 \mathrm{~mm}$. For $r=100 \%$, the TIR-QPM in a hexagonal cavity greater than that from PPM method for an equivalent length while for $r=99 \%$ degrades down toward that of NPM. 
In equation (4) the input $I_{\omega}$ is not a constant value up to until 13 round-trips but takes the values of FW from figure 7 for TIR-QPM. For PPM and QPM, $I_{\omega}$ was constant and equal to the starting $I_{\omega}$ used for TIR-QPM because there is no resonance of the FW in these conditions. The SH wave was allowed to propagate until it reached the steady state, after which no further build-up is occurred. After 13 round trips, the growth in $\mathrm{SH}$ is mostly due to the resonance of the $\mathrm{SH}$ but not the conversion from FW. $I_{\omega}$ is amplified after each of the round trips by the resonance of FW within the cavity. This amplification, boosts the SH efficiency (via the TIR-QPM) to be more efficient than that via PPM and QPM methods. Without this amplification, TIR-QPM method will never be more efficient than the PPM method, and for our choice of the cavity size $\alpha_{\mathrm{m} 2}=13.95 \mu \mathrm{m}$, it will be less efficient as compared to the QPM method (see figure 2). The total length of the devices used to estimate the SH growth for $P P M$ and $Q P M$ is $N L=3 N \alpha=1.25 \mathrm{~mm}$, about 90 time longer than the hexagonal microcavity used for TIR-QPM.

The effective number of reflections inside the microcavity is limited by the Goos-Hanchen shift and by the surface roughness on the sidewalls of the cavity which controls the value of $r$ [18]. There is an effective number of single bounces $\mathrm{M}$ or equivalently of corresponding number $\mathrm{N}$ of round trips for $\mathrm{a}$ given reflectivity $r$ after which there will be no wave's build-up as can be derived by the asymptotic behaviour of the following trigonometric factor of equation (4):

$$
A=\frac{r^{M}}{M^{2}} \frac{1-2 \sqrt{r^{M}} \cos (M \phi)+r^{M}}{1-2 \sqrt{r} \cos (\phi)+r}
$$

where $\phi$ is the global phase shift given by Eq. (3). The effect of reflectivity $r$ is shown in figure 9 where $I_{2 \omega}$ drops as the reflection losses increase.

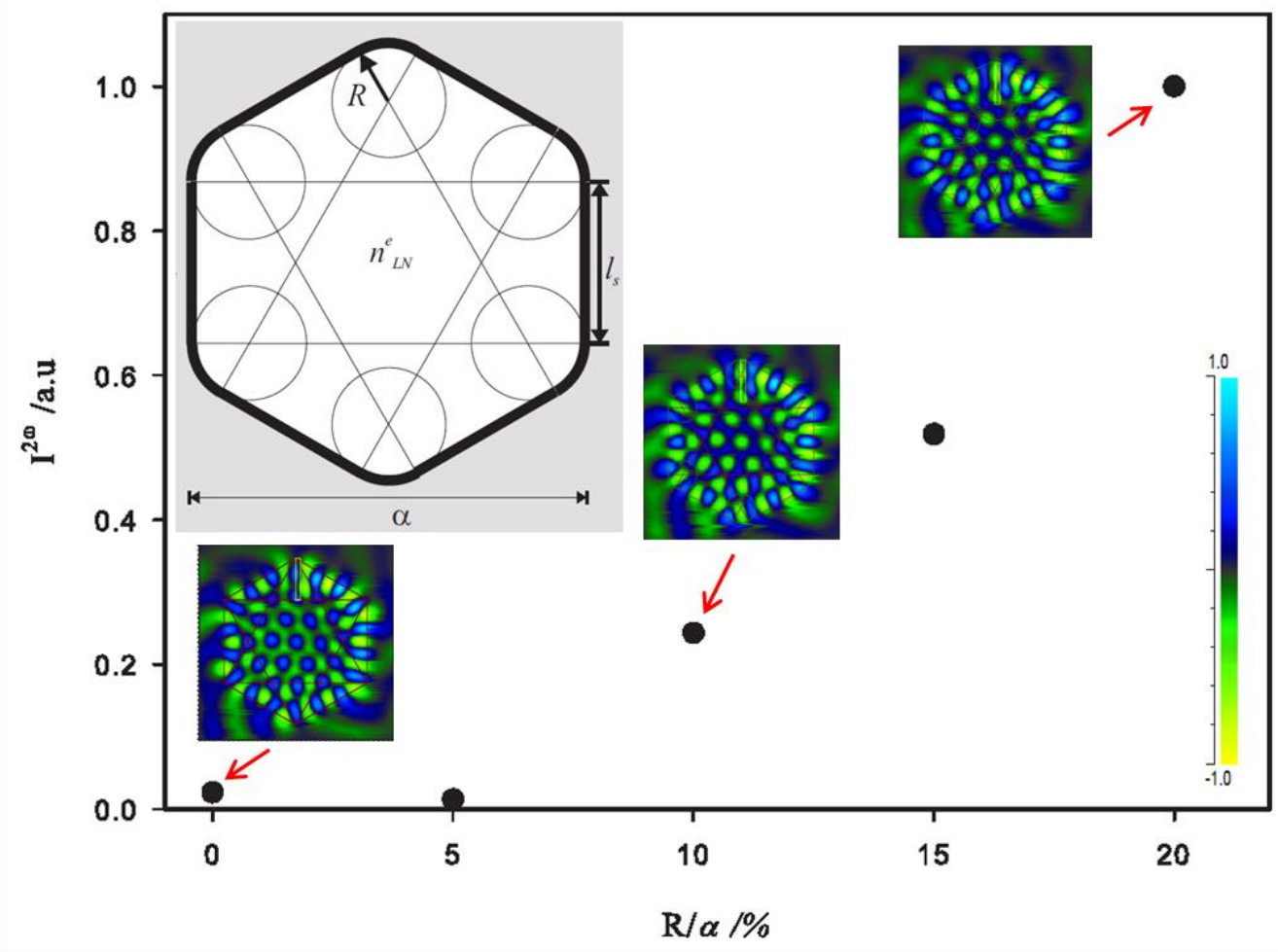

Figure 10: Growth of the SH for different degrees of rounding of the hexagonal corners, for $\lambda=0.955 \mu \mathrm{m}$ with $\alpha=13.95 \mu \mathrm{m}$. The corresponding steady state modeled patterns illustrate the scattering effect at the corners of the cavity as attributed to the effect of rounding. 
It was studied also the optimization of hexagonal cavity's shape by considering the effect of corners rounding. The degree of rounding is described by the parameter $R / \alpha$ where $R$ is the local radius of the curvature of the circle that induces the rounding in the hexagon and varies from 0 to $0.5 \alpha$ corresponding to hexagonal and circular microcavities respectively. As R/ $\alpha$ increases, the $Q$ factor increases, leading to longer cavity lifetime for both the FW and the SH and hence to SH efficiency increase. By increasing $\mathrm{R} / \alpha$ ratio this results in a blue-shift in the resonance wavelength, while this can be attributed to the growth of the cavity size per rounding resulting in a longer cavity path length. Hence, to keep the resonating wavelength constant, the cavity size has to be reduced per rounding for the cavity path length to remain constant. As the employed TIR-QPM model does not see any rounding effects applied to the cavity within the six-bounce trajectories, we keep the wavelength and cavity size constant and use the rounding-effect results.

Figure 10 shows the estimated normalized SH signal as related to the degree of cavity rounding. The inset colour images illustrate indicatively different steady state resonance of $\mathrm{SH}$ for different degrees of rounding, demonstrating also the decreased scattering effect at the rounded corners' compared to non-rounded corners in the typical hexagonal cavity. The SH wave increases with smoothing of the corners of the cavity owing to the increasing $Q$-factor at resonance. The rounding effect for $(R / \alpha)>20 \%$ is not shown as the propagation mechanism within the cavity changes from a six-bounce trajectory to whispering gallery type.

\section{Conclusions}

Microresonators are key components for high density integrated optical circuits suitable for a variety of applications, from sensing, to high channel count optical filters for DWDM systems. In addition the integration of nonlinear optical resonators, in integrated optical circuits is expected to greatly enhance their overall functionality and improve their role in more demanding applications. The proposed scheme for SHG via TIR-QPM in a hexagonal microcavity can improve the efficiency and also the compactness of SHG devices compared to traditional linear-type based devices. A simple theoretical model based on six-reflection trajectory and phase matching conditions of the FW and $\mathrm{SH}$ via the TIR-QPM technique was capable for obtaining simple design rules for the determination of optimal cavities. Furthermore numerical simulation results based on FDTD BPM analysis confirmed the solutions obtained by demonstrating resonant operation of the microcavity first in the FW and also in the SH wave produced by TIR-QPM. These results suggest the feasibility of this proposed scheme that could also be extended to higher order polygons.

The efficiency of the proposed TIR-QPM method is shown to be limited mostly by the reflection losses which limits the number of round trips to an effective number of round trips and hence compromises the build-up of the SH per cavity round. However TIR-QPM is capable of a high SH efficiency, even greater than that of perfect phase matching, for the ideal case of $100 \%$ reflectivity, due to the simultaneous resonance of both the FW and SH within the same cavity. The loss induced by the sharp corners which is a common problem in polygonal resonators and can degrade also the performance of passive devices could be overcome by the use of smoothed round cornered micro cavities, as it has been shown that the associated improvement in the $Q$ factor and finesse can lead to drastical improvement in their operational characteristics. Further work is currently in progress in order to consider improved and more realistic FDTD simulation methods, combined also with 
rigorous multi-parametric optimization strategies [37] for the overall improvement of nonlinear microresonators performance.

Lithium Niobate can serve as a useful material platform to demonstrate and implement effectively the proposed SHG scheme because of its high non-linearity and its preferred hexagonal shaped resonators which result during chemical etching of the inverted ferroelectric domains. Furthermore current techniques of direct laser writing of waveguides in Lithium Niobate can be combined for the hybrid integration of different linear and non-linear components in multifunctional optical circuits.

Acknowledgements: T.J.S. gratefully acknowledges the Commonwealth Scholarship Commission of the United Kingdom for financial support.

\section{References}

[1] K. J. Vahala, "Optical microcavities," Nat., vol. 424, p. 839, 2003.

[2] K. J. Vahala, ed., Optical Microcavities, vol. 5, Advanced series in Applied Physics. World Scientific, 2004.

[3] D. Klunder, E. Kriosukov, F. S. Tan, T. V. D. Veen, H. F. Bulthuis, G. Sengo, C. Otto, H. J.W. M. Hoekstra, and A. Driessen, "Vertically and laterally waveguide coupled cylindrical microresonators in Si3N4 on SiO2 technology," Appl. Phys. B, vol. 73, p. 603, 2001.

[4] J. Wiersig, "Hexagonal dielectric resonators and microcrystal lasers," Phys. Rev. A: At. Mol. Opt. Phys., vol. 67, p. 023807, 2003.

[5] X. Wang, Q. Liao, X. Lu, H. Li, Z. Xu, H. Fu, "Shape-engineering of self-assembled organic single microcrystal as optical microresonator for laser applications," Scientific reports, 4:7011, 2014

[6] B. Little, S. Chu, H. Haus, J. Foresi, and J.-P. Laine, "Microring resonator channel dropping filters," J. Lightwave Technol., vol. 15, p. 998, 1997.

[7] B. Little, W. Chu, S.T.and Pan, D. Ripin, T. Kaneko, Y. Kokubun, and E. Ippen, Vertically coupled glass microring resonator channel dropping filters," IEEE Photon. Technology. Lett., vol. 11, no. 2, p. 215, 1999.

[8] V. Van, T. A. Ibrahim, P. P. Absil, F. G. Johnson, R. Grover, and P. T. Ho, "Optical signal processing using nonlinear semiconductor microring resonators," IEEE J. Sel. Top. Quantum Electron., vol. 8, no. 3, p. 705, 2002.

[9] D. V. Strekalov, C. Marquardt, A. B. Matsko, H. G. L. Schwefel, and G. Leuchs, "Nonlinear and quantum optics with whispering gallery resonators," Journal of Optics, vol. 18, no. 12, 123002, 2016

[10] C. Li, L. Zhou, S. Zheng, and A. W. Poon, "Silicon polygonal microdisk resonator," IEEE J. Sel. Top. Quantum Electron., vol. 12, no. 6, p. 1438, 2006.

[11] A. K. Bhowmik, "Polygonal optical cavities," Appl. Opt., vol. 39, p. 3071, 2000.

[12] C. Li and A. W. Poon, "Experimental demostration of waveguide-coupled round cornered octagonal microresonator in silicon nitride," Opt. Lett., vol. 30, no. 5, p. 546, 2005.

[13] T. Kouno, M. Sakai, K. Kishino, and K. Hara, "Optical microresonant modes acting in thin hexagonal GaN microdisk," Japanese Journal of Applied Physics vol. 53, 072001, 2014.

[14] S. Mailis, C. Riziotis, P.G.R. Smith, J.G. Scott, R.W. Eason, "Continuous wave ultra violet radiation induced frustration of etching in lithium niobate single crystals", Applied Surface Science, vol. 206, no. 1 p. 46, 2003.

[15] S. Mailis, C. Riziotis, I.T. Wellington, P.G.R. Smith, C.B.E. Gawith, R.W. Eason, "Direct ultraviolet writing of channel waveguides in congruent lithium niobate single crystals", Optics Letters, vol. 28, no. 16, p.1433, 2003.

[16] K. Kalli, C. Riziotis, A. Posporis, C. Markos, C. Koutsides, S. Ambran, A.S. Webb, C. Holmes, J.C. Gates, J.K. Sahu, P.G.R. Smith, "Flat fibre and femtosecond laser technology as a novel 
photonic integration platform for optofluidic based biosensing devices and lab-on-chip applications: current results and future perspectives," Sensors and Actuators B. Chemical, 209, p. 1030, 2015.

[17] P. P. Absil, J. V. Hryniewicz, B. E. Little, P. S. Cho, R. A. Wilson, L. G. Joneckis, and P.-T. Ho, "Wavelength conversion in GaAs micro-ring resonators," Opt. Lett., vol. 25, no. 8, p. 554, 2000.

[18] V. S. Ilchenko, A. A. Savchenkov, A. B. Matsko, and L. Maleki, "Nonlinear optics and crystalline whispering gallery mode cavities," Phys. Rev. Lett., vol. 92, pp. 043903-1, 2004.

[19] X. Guo, C-L. Zou, H.X. Tang, Second-harmonic generation in aluminum nitride microrings with 2500\%/W conversion efficiency, Optica vol. 3, no. 10, p. 1126, 2016

[20] Z. Lin, X. Liang, M. Lončar, S. G. Johnson, A.W. Ropdriguez, "Cavity-enhanced secondharmonic generation via nonlinear-overlap optimization", Optica, vol. 3, no. 3, p. 233, 2016.

[21] T. J. Sono, J. G. Scott, C. L. Sones, C. E. Valdivia, S. Mailis, R. W. Eason, J. G. Frey, and L. Danos, "Reflection second harmonic generation on a z-cut congruent lithium niobate crystal," Phys. Rev. B: Condens. Matter, vol. 74, 205424, 2006.

[22] J. A. Armstrong, N. Bloembergen, and J. D. P. S. Pershan, "Interaction between light wave in a nonlinear dielectric," Phys. Rev., vol. 127, no. 6, p. 1918, 1962.

[23] M. M. Fejer, G. A. Magel, D. H. Jundt, and R. L. Byer, "Quasi-phase-matched second harmonic generation: Tuning and tolerances," IEEE J. Sel. Top. Quantum Electron., vol. 28, no. 11, p. 2631, 1992.

[24] T. J. Sono, "The study of surface SHG and polygonal microcavity design for nonlinear applications on $\mathrm{LiNbO}_{3}$," Optoelectronics Research Centre, Faculty of Engineering, Science and Mathematics, University of Southampton, PhD Thesis, 2009.

[25] S. Banik, U. Das, S. Deb, and A. Saha, "Numerical analysis of broadband second harmonic generation using TIR-QPM in a parabolic profiled isotropic semiconductor slab," Optics Communications, vol. 295, p. 180, 2013.

[26] C. Riziotis, T. J. Sono, S. Mailis, and R. W. Eason, "Enhanced second harmonic generation in lithium niobate hexagonal micro-resonator via total internal reflection quasi-phasematching," Proc. of SPIE 8964, Nonlinear Frequency Generation and Conversion: Materials, Devices, and Applications XIII, 89641Q, 2014.

[27] H. Komine, W. H. Long, J. W. Tully, and E. A. Stappaerts, "Quasi-phase-matched secondharmonic generation by use of total-internal-reflection phase shift in gallium arsenide and zinc selenide plates," Opt. Lett., vol. 23, no. 9, p. 661, 1998.

[28] R. Haidar, N. Forget, P. Kupecek, and E. Rosencher, "Fresnel phase matching for three-wave mixing in isotropic semiconductors," J. Opt. Soc. Am. B: Opt. Phys., vol. 21, no. 8, p. 1522, 2004.

[29] R. Haidar, "Fractional quasi-phase-matching by Fresnel birefrigence," Appl. Phys. Lett., vol. 88, p. 211102, 2006.

[30] N. G. R. Broderick, G. W. Ross, H. L. Offerhaus, D. J. Richardson, and D. C. Hanna, "Hexagonally poled lithium niobate: a two-dimensional nonlinear photonic crystal," Phys. Rev. lett., vol. 84, no. 19, p. 4345, 2000.

[31] C. L. Sones, S. Mailis, W. S. Brocklesby, R. W. Eason, and J. R. Owen, "Differential etch rates in z-cut LiNbO3 for variable HF/HNO3 concentrations," J. Mater. Chem., vol. 12, p. 295, 2002.

[32] R. C. Miller, "Optical second harmonic generation in piezoelectric crystals," Applied Physics Letters, vol.5 no.1, p.17, 1964.

[33] P. D. Maker, R. W. Terhune, M. Nisenoff, and C. M. Savage, "Effects of dispersion and focusing on the production of optical harmonics", Phys. Rev. Lett. vol. 8, p.21, 1962.

[34] N. Ma, C. Li, and A. W. Poon, "Laterally coupled hexagonal micropillar resonator add drop filters in silicon nitride," IEEE Photon. Technol. Lett., vol. 16, no. 16, p. 2487, 2004. 
2

4

5

6

7

8

9

10

11

12

13

14

15

16

17

18

19

20

21

22

23

24

25

26

27

28

29

30

31

32

33

34

35

36

37

38

39

40

41

42

43

44

45

46

47

48

49

50

51

52

53

54

55

56

57

58

59

60

[35] N. Ma, Laterally coupled hexagonal micro-pillar resonator add-drop filter for optical communications. MPhil, The Hong Kong Univ. of Science and Tech., 2004.

[36] N. Ma, F. K. L. Tung, S. F. Lui, and A. W. Poon, "Hexagonal micro-pillar cavities: multimode resonances and open-loop resonance linewidth broadening," in Proc. SPIE 4986, Physics and Simulation of Optoelectronic Devices XI, 153, 2003.

[37] C. Riziotis, A. Vasilakos, "Computational intelligence in photonics technology and optical networks: A survey and future perspectives," Information Sciences, vol. 177, no. 23, p. 5292, 2007. 